ИЗВЕСТИЯ АКАДЕМИИ НАУК ЭСТОНСКОИ ССР. ФИЗИКА * МАТЕМАТИКА

PROCEEDINGS OF THE ACADEMY OF SCIENCES OF THE ESTONIAN SSR.

PHYSICS * MATHEMATICS

$1989,38,1$

удК 535.55

С. ИДНУРМ

\title{
ИНТЕГРАЛЬНАЯ ФОТОУПРУГОСТЬ ДИСКА ГЕРЦА
}

\author{
(Представил Х. Абен)
}

\section{1. Введение}

Задача круглой пластины, которая сжимается двумя сосредоточенными силами, направленными по ее диаметру, имеет хорошо известное из теории упругости $\left[{ }^{1}\right]$ теоретическое решение. Поэтому такую задачу (диск Герца) часто используют для проверки различных экспериментальных методов. В поляризационно-оптическом методе обычно этот диск просвечивают перпендикулярно его срединной плоскости. Так как напряжения по толщине диска постоянны (плоская задача), то при помощи метода двумерной фотоупругости можно прямо определить разности главных напряжений и их направления. Численным интегрированием уравнений равновесия можно напряженное состояние определить полностью.

В данной статье рассматривается возможность определения напряжений в диске Герца методом интегральной фотоупругости $\left[{ }^{2}\right]$ просвечиванием диска в его плоскости. Целью работы является выяснение возможности определения неосесимметричного напряженного состояния методом интегральной фотоупругости. Для исключения части из неизвестных коэффициентов в интегральной фотоупругости используются уравнения теории упругости $\left[{ }^{3}\right]$.

\section{2. Использование уравнений равновесия}

Рассмотрим круглый диск, сжатый вдоль диаметра (рис. 1). Найдем напряжения в виде

$$
\begin{aligned}
\sigma_{r} & =\sum_{m, n=0,2, \ldots} a_{m n} Q^{n} \cos m \theta, \\
\sigma_{\theta} & =\sum_{m, n=0,2, \ldots} b_{m n} \varrho^{n} \cos m \theta, \\
\sigma_{r \theta} & =\sum_{m, n=0,2, \ldots} c_{m n} \varrho^{n} \sin m \theta,
\end{aligned}
$$

где $\varrho=r / R$. Коэффициенты $a_{m n}, b_{m n}$ и $c_{m n}$ подлежат определению на основе данных эксперимента.

Подставляя выражения напряжений (1) в уравнения равновесия

$$
\begin{gathered}
\frac{\partial \sigma_{r}}{\partial r}+\frac{1}{r} \frac{\partial \sigma_{r \theta}}{\partial \theta}+\frac{\sigma_{r}-\sigma_{\theta}}{r}=0, \\
\frac{1}{r} \frac{\partial \sigma_{\theta}}{\partial \theta}+\frac{\partial \sigma_{r \theta}}{\partial r}+2 \frac{\sigma_{r \theta}}{r}=0,
\end{gathered}
$$


получим

$$
\begin{aligned}
c_{m n} & =\frac{m}{n+2} b_{m n}, \\
a_{m n} & =\frac{n+2-m^{2}}{(n+1)(n+2)} b_{m n} .
\end{aligned}
$$

При просвечивании диска вдоль линии $l$ (рис. 1), разность хода равняется

$$
\delta=\int_{l} \sigma_{x}^{\prime} d_{y}^{\prime}
$$

где

$$
\sigma_{x}^{\prime}=\sigma_{r} \cos ^{2} \theta^{\prime}+\sigma_{\theta} \sin ^{2} \theta^{\prime}-\sigma_{r \theta} \sin \theta^{\prime} \cos \theta^{\prime} .
$$

Из выражений (1), (3) иі (4) имеем

$$
\begin{aligned}
& \delta=\sum_{m, n=0,2, \ldots} b_{m n}\left\{\frac{n+2-m^{2}}{(n+1)(n+2)} \int_{l} Q^{n} \cos m \theta \cos ^{2} \theta^{\prime} d y^{\prime}+\right. \\
& +\int_{l} \mathrm{Q}^{n} \cos m \theta \sin ^{2} \theta^{\prime} d y^{\prime}-\frac{m}{n+2} \int_{l} \mathrm{Q}^{n} \sin m \theta \sin \theta^{\prime} \cos \theta^{\prime} d y^{\prime} .
\end{aligned}
$$

Учитывая соотношения

$$
\xi=\frac{x^{\prime}}{R}, \quad \eta=\frac{y^{\prime}}{R},
$$

$\cos \theta^{\prime}=\xi / \varrho, \quad \sin \theta^{\prime}=\frac{\sqrt{\varrho^{2}-\xi^{2}}}{\varrho}, \quad y^{\prime}=R \sqrt{\varrho^{2}-\xi^{2}}$,

можно интегралы в выражении (6) переписать в виде

$$
\begin{gathered}
\int_{l} \varrho^{n} \cos m \theta \cos ^{2} \theta^{\prime} d y^{\prime}=2 R \xi^{2} \cos m \beta \int_{\xi}^{1} \frac{\varrho^{n-1} T_{m}(\xi / \varrho) d \varrho}{\sqrt{\varrho^{2}-\xi^{2}}}, \\
\int_{l} \varrho^{2} \cos m \theta \sin \theta^{\prime} d y^{\prime}=2 R \cos m \beta\left\{\int_{\xi}^{1} \frac{\varrho^{n+1} T_{m}(\xi / \varrho) d \varrho}{\sqrt{\varrho^{2}-\xi^{2}}}-\right. \\
\left.-\xi^{2} \int_{\xi}^{1} \frac{\varrho^{n-1} T_{m}(\xi / \varrho) d \varrho}{\sqrt{\varrho^{2}-\xi^{2}}}\right\},
\end{gathered}
$$

$\int_{l} \varrho^{n} \sin m \theta \sin \theta^{\prime} \cos \theta^{\prime} d y^{\prime}=2 \xi R \cos m \beta \int_{\xi}^{1} \varrho^{n-1} S_{m}(\xi / \varrho) d \varrho$,

где $T_{m}(\xi / \varrho)=\cos m \arccos (\xi / \varrho)$ - полином Чебышева,

$S_{m}(\xi / \varrho)=\sin m \arccos (\xi / \varrho)$. виде

Используя полученные интегралы (8), перепишем уравнение (6) в

$$
\begin{aligned}
\delta=2 R & \sum_{m} \cos m \beta \sum_{n} b_{m n}\left\{\int_{\xi}^{1} \frac{\varrho^{n+1} T_{m}(\xi / \varrho) d \varrho}{\sqrt{\varrho^{2}-\xi^{2}}}-\frac{m^{2}+n^{2}+2 n}{(n+1)(n+2)} \xi^{2} \times\right. \\
& \times \int_{\xi}^{1} \frac{\varrho^{n-1} T_{m}(\xi / \varrho) d \varrho}{\sqrt{\varrho^{2}-\xi^{2}}}-\frac{2 m}{n+2} \xi \int_{\xi}^{1} \varrho^{n-1} S_{m}(\xi / \varrho) d \varrho .
\end{aligned}
$$




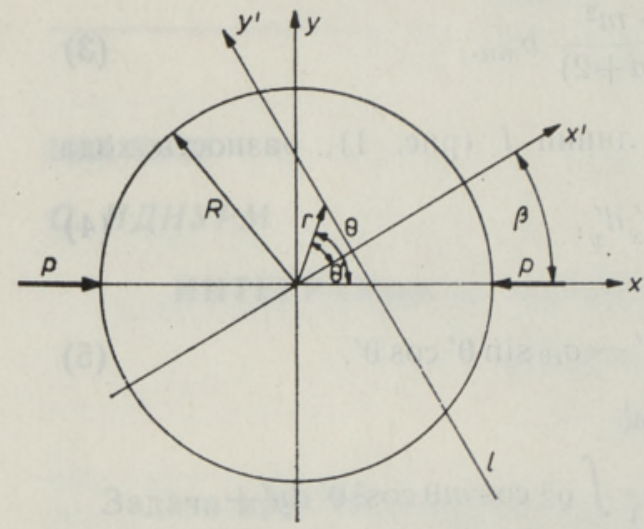

Рис. 1. Расположение координатных осей.

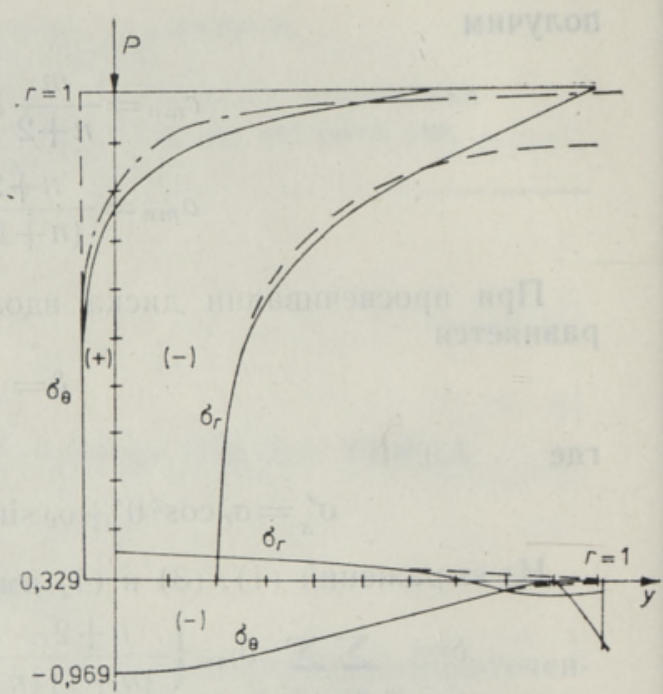

Рис. 2. Графики напряжений (сплошные кривые - эксперименты $(m=18)$; штриховые - теоретическое решение; штрихпунктирные - данные $\left.\left[{ }^{7}\right]\right)$.

Разность хода $\delta$ является функцией от координат $\xi$ и $\beta$

$$
\delta=\delta(\xi, \beta) .
$$

Если разложить $\delta$ в ряд косинусов

$$
\delta=\sum_{m} \delta_{m}(\xi) \cos m \beta,
$$

и поставить полученный ряд в интегральное уравнение (9), то после сравнения коэффициентов одинаковых косинусов, получим

$$
\delta_{m}(\xi)=2 R \sum_{n} b_{m n}\left\{\int_{\xi}^{1} \frac{\varrho^{n+1} T_{m}(\xi / \varrho) d \varrho}{\sqrt{\varrho^{2}-\xi^{2}}}-\right.
$$

$\left.-\frac{m^{2}+n^{2}+2 n}{(n+1)(n+2)} \xi^{2} \int_{\xi}^{1} \frac{\varrho^{n-1} T_{m}(\xi / \varrho) d \varrho}{\sqrt{\varrho^{2}-\xi^{2}}}-\frac{2 m}{n+2} \xi \int_{\xi}^{1} \varrho^{n-1} S_{m}(\xi / \delta) d \varrho\right\}$.

Сканируя диск под различными углами $\beta$, определяем из (11) величину $\delta_{m}(\xi)$ и для каждого значения $m$ получим для определения неизвестных коэффициентов $b_{m n}$ систему линейных уравнений. Однако, из этих уравнений нельзя определить все значения $b_{m n}$, так как детерминант полученных уравнений равняется нулю.

Например, при $m=0$ получим из (12)

$$
\delta_{0}(\xi)=2 R \sqrt{1-\xi^{2}} \sum_{n=0,2, \ldots} \frac{b_{0 n}}{n+1},
$$

т. е. на основе экспериментальных данных можно определить только сумму

$$
\sum_{n=0,2, \ldots} \frac{b_{0 n}}{n+1}=b_{00}+\frac{b_{02}}{3}+\frac{b_{04}}{5}+\ldots
$$


Таким образом, хотя уравнения равновесия позволяют формально элиминировать из трех групп неизвестных две, получаемая для определения третьей группы неизвестных система уравнений не имеет однозначного решения. Это согласуется с результатами работы $\left[{ }^{4}\right]$, где показано, что при определении тензорного поля с помощью продольных волн надо дополнительно использовать условие совместности деформаций Сен-Венана.

\section{3. Использование уравнения совместности деформаций}

Уравнение совместности деформаций для плоского напряженного состояния можно записать в виде

$$
\left(\frac{\partial^{2}}{\partial r^{2}}+\frac{1}{r} \frac{\partial}{\partial r}+\frac{1}{r^{2}} \frac{\partial^{2}}{\partial \theta^{2}}\right)\left(\sigma_{r}+\sigma_{\theta}\right)=0 .
$$

Подставляя уравнения (1) и (3) в (15), получим

$$
\frac{\left((n+2)^{2}-m^{2}\right)\left(n^{2}-m^{2}\right)}{(n+1)(n+2)} b_{m 2 Q^{n-2} \cos m \theta=0 .}
$$

Из соотношения (16) вытекает, что для удовлетворения уравнения совместности деформаций должно быть выполнено условие

$$
\left((n+2)^{2}-m^{2}\right)\left(n^{2}-m^{2}\right)=0,
$$

или

$$
\begin{aligned}
& n= \pm m-2 \\
& n= \pm m .
\end{aligned}
$$

Отсюда видно, что для каждого значения $m$ можно иметь только два положительных значения $n$, которые удовлетворяют уравнению совместности деформаций. Коэффициенты, у которых $n<0$, должны равняться нулю, так как напряжения в центре диска имеют конечное значение. Для случая $m=0$ имеет смысл только случай $n=m=0$, т. е. все члены кроме $b_{00}$ не удовлетворяют уравнению совместности деформаций и они должны равняться нулю. В случае, когда напряжения аппроксимируются тригонометрическими рядами (1), придется использовать только те члены, которые удовлетворяют условию (18). Такие же члены использованы в обобщенном решении двумерной задачи в полярных координатах $\left[{ }^{5}\right]$.

\section{4. Численный эксперимент}

При помощи условия равновесия сегмента [ $\left.{ }^{6}\right]$ были генерированы теоретические экспериментальные данные для 10 значений угла в 20 точках линии сканирования

$$
\begin{array}{ll}
\delta=P \cos \beta, & \left(x^{\prime} \leqslant R \cos \beta\right) ; \\
\delta=0, & \left(x^{\prime}>R \cos \beta\right) .
\end{array}
$$

Напряжения аппроксимировали рядами (1), используя только члены $m=n$ и $n=m-2$. Максимальное значение $m=18$. Полученные системы решали методом наименьших квадратов. На рис. 2 приведены графики напряжений $\sigma_{r}$ и $\sigma_{\Theta}$. Как видно из графиков, они довольно точно совпадают с теоретическим решением (кроме граничных точек). Причиной несовпадения на границах является обстоятельство, что сосредоточенную силу $P$ трудно точно разложить в ряд косинусов (11). На рис. 2 приведены еще результаты экспериментов из работы [ $\left.{ }^{7}\right]$, которые довольно хорошо совпадают с эпюрой напряжения при $m=18$. 
1. Демидов С. П. Теория упругости. М., Высш. шк., 1979.

2. Абен X. K. Интегральная фотоупругость. Таллин, Валгус, 1978.

3. Aben, H., Idnurm, S. Integrated photoelasticity of the general three-dimensional stress state // Proc. of the Int. Conf. «Measurement of static and dynamic parameters of structures and materials». Plzen, 1987, 1, 9-16.

4. Шарафутдинов В. А. // Сибирский мат. ж., 1983, 24, № 6, 176-218.

5. Тимошенко С. П., Гудьер Дж. Теория упругости. М., Наука, 1975.

6. Poritsky, H. // Physics, 1934, 5 (December), 406-411.

7. Pindera, J. T. // Opt. Eng., 1982, 21, № 4, 672-678.

Ннститут кибернетики

Академии наук Эстонской ССР
Поступила в редакцию $12 / 1 \quad 1988$

\section{S. IDNURM}

\section{HERTZI KETTA INTEGRAALNE FOTOELASTSUS}

On vaadeldud pingete määramist piki diameetrit kahe koondatud jõuga surutud kettas integraalse fotoelastsusmeetodi abil, valgustades ketast tema pinnas. Pinged on aproksimeeritud trigonomeetriliste ridade (1) abil. Osa tundmatuid kordajaid pingete avaldustes on elimineeritud tasakaaluvôrrandite (2) abil ning näidatud, et kuigi tasakaaluvõrrandid võimaldavad elimineerida kolmest tundmatute grupist kaks (3), ei ole kolmanda grupi tundmatute määramiseks tekkinud lineaarvôrrandite süsteemil (12) ühest lahendit. Lahendi saamiseks tuleb kasutada pingete avaldistes ainult neid liikmeid (18), mis rahuldavad deformatsioonide pidevuse tingimust.

On esitatud numbrilise eksperimendi tulemused.

\section{S. IDNURM}

\section{INTEGRATED PHOTOELASTICITY OF THE HERTZ DISC}

Determination of stresses by integrated photoelasticity in a disc loaded along a diameter by concentrated forces is considered. The stresses are approximated by expansions (1). Two groups of unknown coefficients (3) are eliminated by the equations of equilibrium. For determination of the third group coefficients we get an overdetermined system of linear equations (12). It is possible to solve this system using only coefficients (18) in the expansions of stresses which satisfy the equation of compatibility.

The results of a numerical experiment are presented. 